\title{
Les étudiants tchèques et slovaques en mobilité internationale : différentes interactions en Erasmus
}

\author{
Marie Červenková, Hana Delalande
}

\begin{abstract}
Les programmes de mobilité internationale permettent aux étudiants de découvrir non seulement un autre système universitaire et d'approfondir leurs connaissances et compétences en langues étrangères mais par le biais de ces séjours ils peuvent connaître d'autres cultures ainsi que leurs porteurs. Les jeunes diplômés ayant effectué une mobilité internationale sont appréciés par les employeurs potentiels sur le marché du travail puisque leurs expériences représentent des atouts supplémentaires de compétitivité : ils font preuve d'une plus grande autonomie, d'une plus grande capacité à prendre des initiatives, de responsabilité et de capacité à résoudre des problèmes.
\end{abstract}

Dans notre contribution nous présentons une recherche en cours de réalisation et ses résultats partiels portant sur la mobilité Erasmus + des étudiants tchèques et slovaques de l'Université Masaryk de Brno. La recherche s'appuie sur des travaux et articles sur le sujet (Papatsiba, 2003 ; Dervin \& Byram, 2008; Parpette \& Mangiante, 2010 ; Murphy-Lejeune, 2013) dont les auteurs examinent les expériences d'étudiants étrangers en France et dans d'autres pays européens. A partir d'un échantillon de participants constitué d'étudiants de la faculté d'Economie et d'Administration qui ont effectué leur mobilité Erasmus+ au cours de 2019 et 2020 dans un pays francophone, nous avons obtenu des données sous forme d'entretiens semi-directifs réalisés après leur retour. L’analyse des données a permis de dégager différents types de difficultés et les interactions des étudiants dans des situations variées non seulement avec les jeunes de leur âge et de même statut mais également avec leurs enseignants, responsables et des employés de bureaux ou d'administration publique avant et aussi pendant la crise sanitaire du Covid 19.

La recherche sur le sujet se poursuivra dans les mois prochains en vue de préparer mieux les étudiants à la mobilité internationale, de les motiver d'une façon adéquate à ce type de séjour et d'adapter les contenus didactiques de nos cours afin d'éviter ou diminuer le choc culturel.

Mots-clés : en français : Erasmus, mobilité, analyse des besoins, interactions, préparation

Abstract : International mobility programs allow students to not only explore a different university system and enhance their foreign languages skills, but through these stays they enable participants to learn about other cultures as well as their bearers. Young graduates who took part in a mobility stay are valued by potential employers on the labor market since their experience represents an additional competitive asset: they demonstrate greater autonomy, better ability to take initiative, responsibility, and deal with problems.

The paper presents an ongoing research and its partial results on Erasmus+ mobility of Czech and Slovak students of Masaryk University in Brno. The research is based on works and articles on the subject (Papatsiba, 2003; Dervin \& Byram, 2008; Parpette \& Mangiante, 2010; Murphy-Lejeune, 2013) whose authors examine the experience of foreign students in France and other European countries. Based on a sample of participants made up of students of the 
Faculty of Economics and Administration who participated in their Erasmus mobility between 2019 and 2020 in a French-speaking country, we obtained data in the form of semi-structured interviews conducted after their return. Data analysis identified different types of difficulties and interactions students encountered in various situations not only with their peers but also their teachers and administrative employees before and also during the Covid 19 health crisis.

The research on the subject will continue in the coming months aiming to better prepare students for international mobility, to motivate them adequately for this type of stay and to adapt the didactic content of language courses in order to avoid or decrease culture shock.

Key words: Erasmus, mobility, needs analysis, interactions, preparation

\section{Introduction}

Les programmes de mobilité internationale permettent aux étudiants de découvrir non seulement un autre système universitaire et d'approfondir leurs connaissances et compétences en langues étrangères mais aussi de connaître, par le biais de ces séjours, d'autres cultures ainsi que leurs porteurs.

Les jeunes diplômés ayant effectué une mobilité internationale sont appréciés par les employeurs sur le marché du travail puisque leurs expériences représentent des atouts supplémentaires en terme de compétitivité : ils font preuve d'une plus grande autonomie, d'une plus grande capacité à prendre des initiatives, de responsabilité, de capacité à résoudre des problèmes.

Pourtant, le nombre d'étudiants tchèques partant en Erasmus diminue. Selon les statistiques de la DZS (Maison de la coopération internationale), le nombre d'étudiants des universités tchèques qui partent à l'étranger en stage Erasmus diminue, les chiffres des étudiants partant en France ou dans d'autres pays francophones descendent $^{1}$ :

$$
\begin{aligned}
& \text { 2014/2015:604 (France) - } 260 \text { - (Belgique) - } 5 \text { (Luxembourg) - } 6545 \text { (total) } \\
& \text { 2015/2016 : } 539 \text { (France) - } 219 \text { - (Belgique) - } 8 \text { (Luxembourg) - } 6290 \text { (total) } \\
& \text { 2016/2017 : } 499 \text { (France) - 174 - (Belgique) - } 8 \text { (Luxembourg) - } 5869 \text { (total) } \\
& \text { 2017/2018: } 419 \text { (France) - } 193 \text { - (Belgique) - } 7 \text { (Luxembourg) - } 5467 \text { (total) }
\end{aligned}
$$

Pour pouvoir réagir à cette situation et stimuler la motivation des étudiants à partir en Erasmus, nous organisons, depuis 2018, des workshops avec des présentations des étudiants alumnis, des discussions avec d'autres étudiants avec la participation des représentants de Campus France République tchèque. Pour pouvoir mieux assister et accompagner les étudiants dans la préparation de leur mobilité, un cours spécifique dédié à la thématique de la mobilité universitaire et

\footnotetext{
${ }^{1}$ Source : https ://www.naerasmusplus.cz/cz/mobilita-osob-vysokoskolske-vzdelavani/statistiky/
} 
la préparation linguistique et interculturelle des étudiants avant le départ est prévu d'être créé. Pour pouvoir rendre ces enseignements les plus efficaces possible, nous nous sommes engagées dans une analyse des besoins de ces étudiants grâce à des entretiens portant sur leur expérience.

\section{Théorie}

La thématique de la mobilité étudiante a suscité l'intérêt de chercheurs différents qui, dans leurs travaux, analysent de façon détaillée l'expérience des étudiants en séjour dans une autre culture que la leur. Les différents sujets qui sont traités sont par exemple les motivations, mais aussi les obstacles que les étudiants rencontrent (Perez-Encinas et al., 2020), les spécificités de l'appréhension de l'étranger, le développement de l'altérité et le processus d'adaptation ou encore le choc culturel (Murphy-Lejeune, 2013; Papatsiba, 2003). Sont traités également les questions de la vie universitaire : les méthodes de travail et les examens, l'insertion des étudiants étrangers et leur accompagnement. Dans certains ouvrages, les chercheurs mettent en évidence le manque de préparation linguistique, méthodologique et interculturelle des étudiants. Ils soulignent le besoin de préparer les étudiants à des pratiques culturellement différentes : prise de notes, écrits universitaires, mais aussi à l'expérience interculturelle en développant la compétence et la sensibilité interculturelles des futurs étudiants Erasmus (Dervin \& Byram, 2008 ; Jackson, 2011; Papatsiba, 2003).

Dans le contexte de la didactique de la langue française étrangère, la nécessité de la formation et préparation des étudiants étrangers qui arrivent dans les universités françaises et francophones a donné naissance à un nouveau concept, le FOU, français sur objectif universitaire (Mangiante \& Parpette, 2011). Dans le dispositif de préparation aux études en français, il est ainsi proposé d'adapter la démarche FOS (Français sur objectif spécifique) et d'analyser les besoins de ces étudiants, de collecter des données en faisant des entretiens ou en analysant les documentations des universités françaises, d'analyser ces données pour enfin en élaborer un cours (Goes \& Mangiante, 2010, p. 145-146). Selon les auteurs, il existe un manque de préparation des étudiants allophones avant leurs départs dans les universités françaises ou francophones, tandis que des moyens institutionnels et pédagogiques devraient être mis en place pour accompagner les étudiants, faciliter leur insertion dans les universités étrangères et augmenter leurs chances de réussir leurs stages d'études (Papatsiba, 2003; p. 256-266). Pour combler ce manque, il est possible d'utiliser des ouvrages méthodologiques spécialisés dans des domaines d'études différents selon les profils des étudiants, par ex. en économie et gestion (Parpette \& Stauber, 2014).

En réaction à un manque de formation (et de motivation) pour la mobilité internationale identifié chez nos étudiants en français des affaires à l'Université Masaryk, 
nous nous sommes inspirées de la démarche FOS pour effectuer notre propre analyse des besoins des étudiants en mobilité et l'analyse des données recueillies nous permettra de proposer de nouvelles activités ou d'élaborer un nouveau cours de FOU ouverts aux étudiants qui souhaitent partir étudier dans les universités françaises et francophones.

\section{Méthodologie de la recherche}

En ce qui concerne la méthodologie, il s'agit d'une recherche qualitative, conduite sur la base de la théorie ancrée, Grounded Theory Method, une méthode de type inductive typique pour les sciences sociales et introduite dans les années $60 \mathrm{du}$ 20e siècle par les chercheurs américains Glaser et Strauss (1967).

Les buts de la recherche sont d'identifier les besoins des étudiants tchèques et slovaques qui partent en Erasmus dans des pays francophones et d'analyser ces besoins en vue de la création d'un cours préparatoire en amont du stage Erasmus pour mieux préparer les étudiants avant le départ.

L'échantillon de la recherche est composé de quatorze étudiants tchèques et slovaques de la Faculté d'Économie et d'Administration de l'Université Masaryk, âgés de 20 à 24 ans, qui ont effectué leurs stages Erasmus en France ou dans des pays francophones pendant les semestres de printemps 2019, automne 2019 et printemps 2020.

Les données de cette recherche ont été recueillies grâce à quatorze entretiens semi-directifs avec les étudiants, la durée de chaque entretien varie entre 45 et 85 min. Trois entretiens réalisés en présentiel ont été enregistrés au dictaphone, les onze autres entretiens ont été réalisés à distance, par téléphone ou grâce au logiciel MS Teams, entre mai 2019 et juillet 2020.

Concernant les universités d'accueil, il s'agissait de huit universités au total, situées en France (Paris Business School - Ecole de commerce, Grande école de Lille - IESEG School of management, Ecole de management de Normandie à Caen, Montpellier Business School et IUT Perpignan), en Belgique (Université VUB de Bruxelles - Solvay Business School et Université Saint Louis à Bruxelles) et au Luxembourg (Université de Luxembourg).

Après la transcription des enregistrements, nous avons effectué une analyse de contenu, suivie par une catégorisation des thématiques et par la définition des catégories et concepts en accord avec la méthodologie de la théorie ancrée. Dans les chapitres suivants est présentée la thématique centrale consistant en la description et l'analyse de différentes interactions réalisées dans des situations de communication variées. Cette thématique a été choisie par les chercheuses en correspondance avec l'une des sections du colloque international Language Centres at 
a Crossroads : Open Directions for New Generations of Learners, soit Learners and their environment : interactions, roles, strategies ${ }^{2}$.

\section{Analyse des données}

Après avoir réalisé et retranscrit les entretiens avec les étudiants, nous avons identifié tous les types d'interactions communicatives dans lesquelles les étudiants s'étaient engagés : à l'école avec d'autres étudiants, avec les enseignants et les employés de l'administration universitaire, hors de la classe avec leurs camarades, les jeunes de leur âge et d'autres personnes dans les situations de la vie quotidienne et ensuite les interactions dues à l'arrivée de la pandémie du Covid 19 en mars 2020. Les entretiens avec les étudiants ont été menés en tchèque, les étudiants ont parlé de leurs expériences dans leur langue maternelle, c'est-à-dire en tchèque ou en slovaque. Pour les besoins de cet article, nous avons traduit les citations des répondants en français. Pendant la transcription, les données ont été anonymisées, les participants à notre recherche sont désignés ici par R1-R14.

\subsection{Interactions avec les enseignants}

Tous les étudiants effectuant leur mobilité Erasmus, c'est-à-dire une mobilité d'études, sont entrés naturellement en contact avec les enseignants des cours suivis. Selon les propos des étudiants nous pouvons observer que le comportement des enseignants envers les étudiants Erasmus diffère selon le type de programme (anglais ou français) et selon le type d'école (université ou école de commerce). En général, les programmes en anglais étaient plus adaptés aux étudiants étrangers puisque la plupart des étudiants dans ces programmes étaient des étudiants Erasmus. Dans les écoles de commerce, les étudiants sont répartis en classes comptant d'habitude une trentaine de personnes qui ne changent pas au cours du semestre. De ce fait, les enseignants et les étudiants étaient plus proches et leurs relations ressemblaient plutôt à celles des écoles secondaires.

Les interactions avec les enseignants se déroulaient généralement sans problème, les enseignants étaient disponibles, prêts à expliquer de nouveau les points que les étudiants n'avaient pas compris, comme dans l'exemple de R8 : ...le cours Economie de l'environnement, il était donné en français, et il a beaucoup développé mes connaissances, c'est un spécialiste qui l'enseignait, un homme super apprécié dans ce domaine. Moi, j'étais la seule étudiante Erasmus dans ce cours... Je dois dire que ce cours était très bien enseigné et organisé, le prof s'occupait de moi. Après chaque cours, il consacrait une demi-heure à m'expliquer en anglais des choses importantes ... il a vu par exemple que je ne saisissais pas trop le cours, il me l'a expliqué à nouveau

\footnotetext{
2 Le XIVe colloque international de l'association des Centres de Langues Cercles tenu du 10 au 12 septembre 2020 à l’Université Masaryk de Brno, République tchèque.
} 
en anglais, m'a envoyé des articles en anglais. Par contre, certains enseignants ne prenaient pas en considération leur statut d'étudiants Erasmus comme le constate R9 : les enseignants n'accordaient pas beaucoup d'attention aux étudiants Erasmus, disant qu'ils devraient savoir ce qu'il faut faire.

Certains étudiants participant à notre recherche se sont inscrits au programme français et ils étaient les seuls étudiants en classe qui ne maîtrisaient pas le français comme langue maternelle. Quelques-uns ont ressenti des problèmes de compréhension, en particulier au début de leur séjour. R9 dit : ...je ne les comprenais pas beaucoup. Je pense que pendant les quinze premiers jours nous étions très fatiguées surtout à cause du français, c'était difficile de faire attention pendant les trois heures de cours et de prendre des notes en même temps. Mais après, ça s'est amélioré, je ne sais même pas comment, mais après j'ai réalisé que j'arrivais à la maison le soir et que j'avais entendu du français toute la journée et que c'était pas un problème pour moi, je n'avais même pas réalisé que c'était une langue étrangère. R11 a choisi, pour éviter d'éventuels malentendus et des sentiments désagréables pendant la communication, une autre stratégie : ensuite, j'ai commencé à enregistrer ces entretiens avec les profs sur mon portable, parce que parfois nous ne les comprenions pas, nous faisions semblant d'avoir compris parce que c'était embarrassant de poser la même question cinq fois. R1 et R2 étaient au contraire surprises de comprendre relativement bien les professeurs sauf ceux qui ne parlaient pas un français standard ou avaient un fort accent en anglais. R1 dit : moi,j'étais bien surprise de bien comprendre les professeurs en classe, ou plutôt, dans un cours, il y avait un prof et lui, il ne parlait pas du tout correctement, en fait, il utilisait de l'argot, des expressions familières et ça me posait problème, mais sinon, je dois dire que je comprenais vraiment bien. Et R2 ajoute : puis, on avait un cours en anglais et il y avait un prof et son anglais, moi, je ne savais pas des fois qu'il parlait anglais, c'était un accent français tellement fort... A partir de cette expérience, ces étudiantes ont recommandé que ceux qui partent en Erasmus et parlent plus ou moins bien français s'inscrivent au programme français.

Dans certains cas et surtout au début de leur séjour, les étudiants n'arrivaient pas à former des équipes de travail avec d'autres étudiants et les enseignants n'intervenaient pas pour qu'ils ne soient pas exclus. La situation s'est progressivement améliorée au cours du semestre au fur et à mesure que les étudiants connaissaient mieux leurs camarades de classe et quand ils ont été capables de s'organiser eux-mêmes. Les règles requises par les enseignants concernant les retards ou les rafraîchissements pendant les cours étaient considérées comme plus strictes par rapport à l'université tchèque. Certains étudiants ont parlé des enseignants qui ne permettaient aux étudiants en retard d'entrer dans la salle qu'après la pause, et de l'interdiction de manger mais aussi de boire pendant le cours.

Les informations sur les formats, les dates et les résultats des évaluations étaient présentées par les enseignants. Cependant, dans quelques cas, les étudiants les ont 
considérées comme insuffisantes ou pas très claires et par conséquent, ils étaient confus, comme R4 lors de son examen : dans le cours de négociations, c'était pas du tout clair, moi, je ne savais même pas qu'on était en train de passer l'examen. Tous les cours ne finissaient pas à la fin du semestre, certains cours se terminaient un mois plus tôt, par exemple ce cours de négociations. On s'est divisés en binômes et on a fait une négociation devant toute la classe, et suite à ça le prof nous a donné une note, sij'ai bien compris, en tout cas, c'était la dernière chose que nous avons faite et puis nous avons vu les notes finales dans le système.

A cause de ce manque d'informations, deux étudiantes n'ont pas pu participer à un examen. R11 dit : ... les dates, on ne nous les a dites que quelques jours avant l'examen. Ou peut-être que les étudiants réguliers le savaient depuis longtemps, mais personne n'a eu l'idée de nous l'annoncer à nous aussi, et donc pendant la première session nous avons manqué au moins un examen. Si nous avions communiqué plus ou avions écrit tous les deux jours à la coordinatrice, nous l'aurions peut-être appris ... Personne n'a eu l'idée de nous mettre dans le carnet d'adresses électroniques. R1 et R2 ont aussi observé que les enseignants ne donnaient pas autant d'informations sur la forme et sur le contenu des examens que dans leur université d'origine où elles faisaient plus d'entraînement pour les tests en classe. Les étudiants ne recevaient pas souvent de retour sur leur travail ou bien si retour il y avait, le nombre de points obtenus par exemple n'était pas clair, comme dans le cas de R13 : jusqu'à présent, je ne sais pas comment cela a été évalué. Personne à l'université ne nous a expliqué le système d'évaluation. Peut-être qu'ils nous l'expliqueront à la fin ou je demanderais à quelqu'un. Nous avons obtenu des points, mais je ne savais pas si j'avais réussi ou non. J'ai dû attendre que les crédits apparaissent pour savoir si j'avais réussi le cours ou non. (...) Ce qui me manquait, c'était un retour, des commentaires, sur mon travail. C'est peut-être à cause du coronavirus et que tout était en ligne. Mais je dois dire que certains résultats m'ont surprise, je n'ai pas compris comment j'avais pu obtenir autant ou si peu de points...

D'autres difficultés sont apparues, par exemple l'interdiction d'utiliser un dictionnaire pendant l'examen malgré la permission explicite dans le syllabus du cours ou bien l'attribution d'un test en langue anglaise dans un cours du programme français. Quant aux compétences langagières des étudiants en français (à part dans les cours de français), la plupart des enseignants ne les évaluaient pas ou exprimaient plutôt leur soutien ou satisfaction avec les efforts des étudiants étrangers. Toutefois, R9 témoigne de l'attitude d'un enseignant qui s'est comporté d'une façon dépréciative envers sa maîtrise du français : un professeur s'est un peu moqué de nous, c'était bizarre, mais enfin... Sinon, c'était cool, on n'évaluait pas beaucoup la langue, mais parfois oui, et ça m'énervait, parce que... je ne sais pas... ils ont dit par exemple: «Mais votre français...» et moi «Monsieur, moi, je ne suis pas Française, ça ne va pas, je ne peux pas être comme les autres». 


\subsection{Interactions avec d'autres étudiants en classe}

L'analyse des entretiens montre que le caractère des interactions avec d'autres étudiants en classe dépendait de plusieurs facteurs, dont le format et la méthode d'enseignement (cours magistraux - travaux dirigés, travail en équipe - travail individuel), le type de programme (programme français - programme anglais), la personnalité de l'étudiant et la personnalité de ses camarades de classe ou bien le fait de venir en Erasmus seul.e ou avec un.e ami.e de son pays.

R11 et R12 regrettent que la plupart des cours aient été donnés sous forme de cours magistraux consistant en un enseignement purement frontal et souvent en un simple recopiage des informations écrites au tableau. De plus, comme les pauses étaient très courtes entre les cours, elles étaient obligées de se dépêcher après le cours pour arriver à temps au suivant. Dans ces conditions, il était presque impossible de nouer des contacts en classe avec d'autres étudiants. Par contre, les étudiants suivant des cours plus interactifs avaient plus de chance de faire la connaissance de leurs camarades de classe puisqu'ils devaient former des équipes de travail et collaborer sur des projets avec les autres.

Dans les programmes anglais suivis par un nombre plus important d'étudiants Erasmus, les relations se sont plus facilement nouées. En effet, tous les étudiants Erasmus se familiarisaient avec un nouvel environnement, souhaitaient trouver de nouveaux amis et rencontraient plus ou moins les mêmes difficultés dans la vie quotidienne dans un pays étranger. Au contraire, dans les programmes français, il n'y avait souvent qu'un seul étudiant Erasmus, ou deux provenant de la même université d'origine. S'ils étaient venus à deux, ils formaient un groupe à part, et étant partiellement satisfaits quant au besoin de partage et de convivialité ils se sont contentés du statu quo et n'ont pas fait autant d'efforts pour créer de nouvelles relations que s'ils étaient seuls. De l'autre côté, les étudiants du pays voyaient que leurs camarades étrangers se suffisaient, dans une certaine mesure, à eux-mêmes. $\mathrm{R} 9$ dit que les étudiants dans le programme anglais ont trouvé des amis plus facilement parce qu'avec elles, les Français ne parlaient pas beaucoup. Moi, je pense que nous étions deux avec (R7), et si j'étais toute seule, ça aurait été différent. Mais comme nous étions deux, les Français croyaient que nous n'avions pas besoin de parler avec eux, (...) et pour eux, c'est aussi plus facile de bavarder avec leurs copains dans leur langue.

La non-communication de certains étudiants français pendant l'élaboration des projets en équipe a causé l'échec du travail de tous les participants, même de ceux qui avaient fait des efforts. R1 dit qu'en tant qu'étrangère elle ne voulait pas persuader les autres de travailler. Selon elle, la communication n'a pas eu de bons résultats aussi à cause des caractères difficiles des étudiants dans le groupe. Elle était également déçue que les autres étudiants en classe ne prennent pas en 
considération que le français n'était pas sa langue maternelle : Pendant le travail en équipe (...), moi, je ne les intéressais pas, ça ne les intéressait pas que j'étais étrangère et ils ne parlaient pas un français correct et utilisaient plein de mots familiers, et puis quand on discutait quelque chose sur Facebook par exemple, ils utilisaient des abréviations, et nous, avec R2, les regardions et ne savions pas ce que ça voulait dire. R2 ajoute que l'échec a été causé par un autre facteur - une mauvaise gestion du temps : D'habitude, on divisait le travail et on travaillait dessus, mais quelquefois il est arrivé que les étudiants commencent à s'y intéresser la veille du jour de la remise. Et ils ont commencé à communiquer à minuit.

\subsection{Interactions avec d'autres étudiants en dehors de la classe}

Les interactions avec d'autres étudiants en dehors de la classe correspondaient dans une certaine mesure aux relations établies en classe. En effet, la plupart des étudiants ne sont pas arrivés à nouer des relations durables avec leurs camarades de classe français ou francophones tandis que ceux dans les programmes anglais se sont relativement bien intégrés dans la communauté d'autres étudiants Erasmus et sont restés en contact même après la fin du séjour à l'étranger. R2 a déclaré :tout le monde le dit, on va dans un pays étranger mais on ne parle pas avec les gens de ce pays mais plutôt avec les étudiants Erasmus. C'est comme ça dans la plupart des cas et moi, je le comprends, quand je vais à l'école ici, dans mon pays, je ne bavarde pas avec les Erasmus. Quoique, maintenant je le ferai.

Alors que R6 désigne la cuisine de la résidence universitaire comme un point de rencontre possible avec d'autres étudiants où elle a facilement trouvé des amis au début de son séjour, l'expérience de R14 est différente : la première impression de la résidence universitaire n'était pas bonne. C'étaient surtout des Français et des Asiatiques qui y logeaient et ils avaient leurs communautés à eux, ils se retrouvaient entre eux. Il n'y avait qu'une cuisine pour les sept étages et il était compliqué de venir dans la cuisine où les étudiants étaient en groupes. Même si je voulais bavarder avec eux, ça n'a pas trop marché, ils ne nous ont pas acceptées.

La personnalité de l'étudiant et celle de ses camarades de classe était aussi un facteur important. Tandis que certains répondants n'ont pas réussi à sortir de leur zone de confort et à oser faire des erreurs en communiquant, d'autres ont pu créer de nouvelles amitiés. Pour R12 il était difficile de faire connaissance avec les autres. N'aimant pas faire des fautes, elle ne se sentait pas à l'aise en français. De plus, elle ne comprenait pas souvent les étudiants d'origine africaine qui étaient logés, comme elle, à la résidence universitaire et qui n'ont pas essayé d'adapter leur façon de parler (français familier, articulation, vitesse) de manière à ce qu'elle les comprenne mieux. L'utilisation de l'argot et du langage sms par les étudiants français empêchait souvent une bonne compréhension des étudiants tchèques et 
slovaques. R3 avoue que la connaissance du langage sms lui manquait : si j'en connaissais au moins quelques-uns, j’aurais évité plusieurs malentendus.

R11 se considère comme moins sociable, elle ne s'adresse donc pas d'habitude à une personne inconnue en première. Quand elle bavardait avec quelqu'un, c'était donc de l'initiative de celui-ci. Grâce à une étudiante Erasmus et sa capacité à réunir les gens, R3 a pu vivre une expérience tout à fait différente, riche en nouvelles relations : Je crois que c'était grâce à une Allemande, étudiante Erasmus, qui a créé un groupe où il y avait des étudiants Erasmus et ses copains de classe. Et, par un concours de circonstances, c'était une communauté de personnes qui aimaient danser, et à l'école on avait des cours de bachata gratuits et on se rencontrait aussi là. A mon avis, c'était un concours de circonstances qu'il y avait cette excellente teamleader et qu'on avait tous le même intérêt, la danse, (...) et peut-être c'était aussi grâce à l'ouverture d'esprit des Français, parce que c'était des gens qui avaient aussi fait Erasmus ou ils aimaient vraiment voyager. (...) Moi, je communique en français au quotidien, je suis toujours en contact avec ces gens, sur facebook ou on s'appelle. Cette ouverture d'esprit des Français envers les étudiants étrangers qui correspondait à leur attitude envers la mobilité Erasmus en général a été aussi mentionnée par R14 : après, j'ai rencontré quelques Français dans les cours en bloc, en anglais, mais eux, ils étaient amicaux, ils s'intéressaient aux étudiants Erasmus, ils étaient cool. Ils se préparaient eux-mêmes à la mobilité Erasmus ou ils l'avaient déjà effectuée. Le fait pour R3 d'avoir fait partie de ce groupe lui a également permis de perfectionner son français : nous nous sommes mis d'accord au début que nous étions ici pour apprendre le français, nous parlerions donc en français. De plus, il y avait une Espagnole qui ne savait pas parler anglais et nous ne voulions pas l'éliminer de notre groupe. C'était un avantage.

Certains étudiants ont remarqué que leur maîtrise du français facilitait les interactions et contribuait à une résolution plus rapide des problèmes. Comme ça, ils ont même pu aider d'autres étudiants en difficultés comme c'était le cas de R1 : C'était un très grand avantage de pouvoir communiquer en français et ça faisait une très grande différence parce que ceux qui ne savaient pas bien parler français mais qui essayaient de régler des choses en anglais, par exemple à la résidence universitaire, on leur a dit, ça ne va pas, on ne peut pas le faire pour vous, ça n'ira pas et ces filles en étaient bien malheureuses, nous sommes arrivées, j'ai posé la question en français, oui, pas de problème, et tout a bien marché. (...) On vous comprendra (en anglais) mais jamais on ne sera aussi disponibles qu'avec le français.

\subsection{Interaction avec les services administratifs}

Alors que les contacts avec les professeurs étaient dans la plupart des cas en présentiel, avec les services administratifs la communication se déroulait en majeure partie par courriel. Avant leur départ, les étudiants sont entrés en contact notamment avec le service international de l'université étrangère. En général, ces inter- 
actions se sont bien déroulées. Par contre, ce qui a vraiment posé problème, c'était la quantité de documents à remplir et en particulier le manque d'informations sur les cours. Non seulement les informations sur le contenu des cours n'apparaissaient pas sur le site universitaire mais en plus, les listes de cours disponibles pour les étudiants Erasmus étaient incomplètes, voire erronées. R3 dit : j'avais un problème, on m'avait envoyé une autre liste. La liste du semestre d'automne au lieu de la liste du semestre de printemps, et personne ne s'en est inquiété. J'ai même reçu deux listes différentes, après je suis arrivée sur place et aucun cours n'était disponible, donc j'ai tout refait, sur place, j'étais hyper stressée, vous pouvez l'imaginer.

Certaines écoles n'ont pas informé les étudiants Erasmus du stage d'un mois à la fin du semestre de printemps destiné aux étudiants réguliers, et en conséquence, les étudiants Erasmus ont dû terminer leur séjour d'études plus tôt et rendre ensuite une partie de leur bourse.

Les écoles organisent d'habitude quelques jours ou une semaine d'intégration, souvent seulement au début de l'année universitaire, c'est-à-dire au semestre d'automne et pas au semestre de printemps. Cet événement aide beaucoup les nouveaux étudiants dans les démarches administratives - qu'il s'agisse de la création d'un compte bancaire, du paiement de l'assurance ou de l'inscription aux cours choisis, tout se fait sur Internet mais avec l'aide des employés de l'école ou d'autres responsables. Pourtant, il y avait des écoles où les étudiants devaient venir au bureau à chaque fois qu'ils voulaient faire un changement dans leur emploi du temps, et de cette façon, la création de l'emploi du temps était très compliquée et a pris beaucoup de temps (chevauchement des cours, longues queues dans le bureau, etc). R11 parle de ce problème ainsi : même si nous nous sommes inscrites aux cours sur Internet et nous les avions dans nos $L A^{3}$, nous avons dû faire après un tour dans les bureaux de la scolarité des facultés et dire ce que nous souhaitions. Mais nous ne savions pas ce que nous souhaitions parce qu'il n'y avait une liste complète nulle part. Le catalogue qu'on nous a donné ne comprenaient que les cours inscrits par les étudiants Erasmus avant nous. Au final, nous avons appris que nous avions un choix beaucoup plus large et nous avons dî faire ce tour des bureaux de scolarité plusieurs fois et à chaque fois que nous y sommes allées, (...), il y avait toujours une file d'attente et nous ne sommes pas arrivées à accéder au guichet parce qu'il y avait un cours qui commençait déjà et nous avons dû y aller et cela a duré pendant des semaines.

Les interactions avec les employés des services universitaires se poursuivaient souvent par mails après le départ des étudiants parce qu'ils n'avaient souvent que des notes provisoires que le service des relations internationales de leur université d'origine n'acceptait pas. Il est aussi arrivé que ces interactions ne puissent plus

\footnotetext{
${ }^{3}$ learning agreement - contrat d'études
} 
se poursuivre pendant les deux mois d'été étant donné qu'il n'y avait personne dans les bureaux.

Les étudiants Erasmus en France peuvent faire une demande pour la CAF, qui est une forme de soutien financier facilitant le paiement du loyer. Quelquefois, les démarches administratives qu'il fallait faire ont pris beaucoup de temps et l'aide n'a été accordée aux étudiants qu'à la fin de leur séjour, voire après leur retour à la maison. C'était par exemple le cas de R3 qui a essayé, à travers cette expérience, de comprendre et d'accepter cet aspect de la situation considéré comme interculturel : mais la demande de la CAF, cela a aussi duré jusqu'à la fin de mon séjour. Ce n'est qu'à la fin que je l'ai eue. (...) Je recommanderais aux étudiants de rester patients, car moi, je n'en pouvais plus, je ne m'attendais pas à ce qu'il en soit ainsi. Mais ensuite je me suis dit, c'est un pays différent, il y a une telle bureaucratie, je dois l'accepter, et quand je veux les allocations du gouvernement français, ce n'est pas si évident, dans d'autres pays ça n'existe pas du tout, je dois faire des sacrifices pour cela. Et ça m'a beaucoup aidé à la fin, ça m'a payé la moitié des loyers mensuels.

L'étudiante R8 était étonnée que certains services de l'administration française emploient des personnes ne parlant pas anglais. Elle décrit son expérience quand elle a déposé une demande de permis de séjour : nous avons attendu une heure et demie, et quand c'était notre tour, la dame ne parlait pas du tout anglais, mais pas du tout, uniquement le français, je lui ai finalement tout expliqué, mais j'aurais préféré traiter de ce genre de choses administratives en anglais. (...) puis nous avons dû le régler par e-mail, et ça, c'était nul. On leur a écrit en anglais, ils ont répondu en français... ça ne me dérange pas, mais il y a des Américains, par exemple, qui ne parlent pas du tout français.

\subsection{Autres types d'interactions}

En dehors de l'école, pendant leur séjour, les étudiants ont effectué des interactions dans la vie courante, c'est-à-dire en faisant les courses, en voyageant, en cherchant un petit job et en travaillant, en communiquant avec les employés du campus ou avec les médecins en cas de maladie.

Etant donné que la plupart des étudiants communiquaient avec leurs camarades de classe en anglais (sauf quelques-uns qui ont fait leurs études dans le programme français et R3 qui pouvait communiquer en français dans la communauté d'étudiants Erasmus et français dont elle faisait partie - cf. chapitre 4.3), ces situations de communication mentionnées ci-dessus ont permis aux étudiants d'améliorer leurs connaissances et compétences en français. Les étudiants étaient plongés tous les jours dans un milieu francophone, le français les entourait partout. De ce fait, ils ont pu perfectionner en particulier leurs compétences réceptives. R6 dit 
qu'en faisant ses courses au marché et en utilisant les transports publics elle a fait des progrès surtout en compréhension orale. Mais pas seulement. Elle a également trouvé un petit job sur un site Internet et a travaillé comme baby-sitter dans une famille franco-tchèque. Comme ça, elle a été amenée à communiquer aussi en français dans la famille et avec les maîtresses et institutrices des enfants. Pour R13 c'étaient notamment les voyages qui lui ont permis de s'engager dans des situations de communication variées : en voyageant nous avons pu nous entraîner en français, par exemple au restaurant, et ça nous a fait énormément plaisir, avec les hôtes, par exemple à Bordeaux nous étions logées chez un hôte très sympa qui nous a fait visiter la ville. Et en fait, l'utilisation du français était très spontanée. C'était formidable. Nous avons aussi essayé Blablacar et ces choses-là.

R7 est entrée en interaction avec des employés dans la résidence universitaire pour régler des problèmes. Dans le premier cas, c'était à cause d'un départ tardif des copains de classe du campus : la résidence était surveillée 24/24, il y avait un réceptionniste, et c'était un avantage et un inconvénient à la fois. A la fin, je me suis disputée avec lui parce qu'il m'a interdit les visites. Nous avons révisé avec un groupe de français dans notre chambre et nous avons terminé vers onze heures du soir. Normalement je devais les accompagner et leur ouvrir la porte. Mais il faisait tard et eux, ils m'ont dit, mais non, reste ici, on est français, on lui demande de nous laisser passer. Et bien sûr, c'était un énorme problème, il m'a fait venir, en disant que si ce sont mes invités, c'est moi qui dois descendre avec eux et qu'il m'interdit les visites. Et donc, je lui dis, oui, interdisez-le-moi, je suis sur le point de rentrer chez moi. Je m'en foutais déjà. Dans le second cas, elle a dû s'adresser à plusieurs reprises au personnel de la résidence à cause d'une panne d'eau chaude dans sa chambre : je n'ai pas eu d'eau chaude pendant très longtemps. (...) J'ai lutté contre ça très longtemps, en septembre, en octobre, et là, ça ne me dérangeait pas trop, parce qu'il faisait vraiment chaud. (...) Ça a commencé à me gêner à la fin d'octobre, c'était trop pour moi. (...) Après, je l'ai annoncé x fois, on doit répéter et répéter des choses aux Français, donc j'y suis allée autant de fois qu'il fallait pour qu'ils le réparent, et ç'a été à la mi-novembre, je pense.

Ensuite, pendant le semestre de printemps 2020, la pandémie du Covid 19 a causé une situation tout à fait imprévue et les étudiants ont été obligés de faire face à de nouvelles situations de communication avec des nouveaux interlocuteurs.

\subsection{Interactions du printemps 2020}

Le début de la pandémie de la Covid-19 en Europe a influencé et compliqué les stages Erasmus de 5 étudiantes de notre échantillon qui se trouvaient en France dans les villes universitaires de Caen (R10, R11 et R12), Montpellier (R13) et Lille (R14). La nouvelle situation a apporté de nombreuses difficultés, mais la période a été très riche en communications et interactions différentes ou inattendues. 
Même lorsque début mars 2020 la République tchèque s'apprêtait à fermer les établissements scolaires et universitaires, les 5 étudiantes rapportent continuer leurs études comme avant, comme en témoigne $\mathrm{R} 13$ : Les nouvelles venaient de la Tchéquie. En France, tout continuait comme avant. Mais avec la décision de la France de fermer les universités, la position des étudiantes allait changer. Selon R13, tout s'est passé très vite après, les informations changeaient au jour le jour, c'était très difficile de s'orienter dans tout cela. Les étudiantes ont été informées par les bureaux internationaux des universités des mesures en cours et des suites possibles de leurs séjours, comme dans l'exemple de R12 : on recevait des mails, les informations n'étaient pas toujours claires ou sûres, on me demandait d'informer le bureau international sur si je reste ou si je pars, on nous a proposé d'annuler notre Erasmus, mais ce n'était pas vraiment clair si on devait après rendre la bourse ou pas et personne ne savait nous répondre. Sous pression de leurs familles en République tchèque et en Slovaquie, 4 étudiantes ont décidé de partir du jour au lendemain, mais R11 en a décidé autrement : je pensais que le virus allait passer, que je pourrais finir mes études comme prévu. R13 est partie du jour au lendemain, les autres filles aussi, mais moi je voulais prendre mon temps et distribuer mes provisions aux autres étudiants à la cité-U, je ne voulais pas tout laisser juste comme ça.

Pour prendre leurs décisions et organiser leurs départs, les étudiantes ont été en interactions écrites par courriel, ou orales par téléphone, avec différents locuteurs : services universitaires, enseignants, compagnies aériennes ou de bus, les Ambassades tchèques et slovaques pour négocier leurs départs puis chercher le meilleur moyen de transport pour se rendre au point de destination. R10 raconte son départ de France : le jour de mon départ, tous les trains et bus ont été annulés. On a trouvé un taxi avec d'autres étudiants Erasmus, on a payé $300 €$ mais on a partagé les frais. Les changements, les imprévus, les déplacements avec les valises et la peur de rater la dernière connexion mettaient les étudiantes dans un état de stress, comme l'explique R13 : mon vol a été annulé, mais l'Ambassade m'a informée de l'existence d'un bus pour le lendemain. C'était très hystérique, pas beaucoup de temps pour tout préparer. Après, à Paris, je devais prendre un taxi pour aller à l'Ambassade mais je n'arrivais pas à le trouver sur le parking. Au téléphone, je ne comprenais pas l'accent du chauffeur, j'étais super stressée, j'avais peur de rater le bus.

Le départ précipité a apporté des situations de communication et interaction imprévues quand il fallait négocier les départs hors contrat et négocier les remboursements des cautions, comme par exemple R13 : J'ai dû payer 600€ parce que je quittais l'appartement précipitamment, j'ai dû utiliser presque tout ce qui me reste de ma bourse Erasmus pour ce paiement. Ça n'a pas été facile. Même après leurs retours en République tchèque ou en Slovaquie, les étudiantes ont dû s'occuper de la gestion administrative et demander les remboursements des amendes ou 
cautions, les communications avec la CAF ou la fermeture des comptes bancaires. R14, encore au moment des entretiens, a décrit ses soucis avec la banque : le compte n'est encore pas clôturé. Je l'avais ouvert pour pouvoir bénéficier de la CAF. Là j'ai reçu un courriel de la CAF, il faut que je retourne une partie des allocations comme je ne suis plus en France. Mais je n'arrive pas à accéder au compte français, à l'argent. Je ne peux rien faire, il y a de l'argent, mais je n'ai pas de codes, ils ont dû être livrés par la poste à l'adresse française après mon départ. Je ne sais pas comment faire pour le clôturer.

Après la fermeture des universités pendant le semestre de printemps 2020, il est possible de constater un manque considérable d'interactions personnelles remplacées par de la communication à distance, des échanges par courriels ou par téléphone. Les étudiantes ont dû agir de manière flexible et s'adapter aux nouvelles situations imprévues.

Les enseignements de la fin du semestre, après les retours des étudiantes en République tchèque ou en Slovaquie, ont été organisés en ligne. Les examens ont été parfois modifiés, les étudiantes ont été évaluées seulement à partir de leur travail continue en France fait avant la fermeture des universités, comme le témoigne R11 : le cours de phonétique, le prof a décidé que l'évaluation du cours, cela sera juste le contrôle continu de la première moitié du cours. Pour le cours d'anglais, on a modifié les modalités d'examen.

Malgré tout, les 5 étudiantes disent qu'elles ne regrettent pas d'être parties parce que les points positifs de leurs expériences sont plus nombreux que les points négatifs. Elles souhaitent toutes repartir en Erasmus plus tard pour le vivre de façon plus «classique». Elles espèrent aussi que leur expérience Erasmus incomplète pourra être reconnue par les futurs employeurs car elles ont dû, chacune individuellement et à leur façon, gérer des imprévus, prendre des décisions en étant stressées et sortir de leur zone de confort.

\section{Discussion}

Les analyses thématiques présentées dans la partie 4 de cet article nous ont servi de données de base pour l'élaboration d'un cours de préparation à la mobilité internationale. Il est possible de diviser les axes thématiques en trois grandes parties, l'organisation universitaire et administrative, les méthodologies de travail et les différences interculturelles.

Pour avoir plus d'informations et comprendre l'organisation universitaire d'un autre pays, les étudiants seront amenés à effectuer une étude plus approfondie des sites web des universités, des programmes d'études proposés pour les Erasmus en français ou en anglais et les comparer et contraster entre eux. Dans le but de s'entraîner aux futures interactions avec différents locuteurs des admi- 
nistrations différentes, nous voulons nous inspirer des expériences des étudiants de cette recherche et des tâches pratiques ou jeux de rôles. De cette façon, les étudiants pourront s'entraîner à rédiger des courriels de demande d'informations et de relance, à remplir des documents administratifs (par ex. CAF, ouverture d'un compte bancaire). Suite aux expériences négatives de certains étudiants de cette étude avec les clôtures de comptes bancaires, un jeu de rôle pourra aider les étudiants à poser des questions à un conseiller bancaire et s'informer de façon détaillée sur les modalités de clôture du compte sur place (avant le départ) ou à distance (après le retour dans leur pays).

Quant à la méthodologie de travail universitaire, il est possible de s'inspirer des ouvrages existants dédiés à cette thématique (Mangiante \& Parpette, 2011; Parpette \& Stauber, 2014) et travailler, avec les étudiants, sur : la prise de notes, la compréhension des modalités des examens, l'analyse des consignes, la rédaction des commentaires, des synthèses, des comptes rendus ou dissertations ou la préparation d'une présentation orale. Les interactions avec les enseignants et les autres collègues de classe constituent une autre partie du travail à l'université. Les étudiants vont s'entraîner davantage à poser des questions aux enseignants ou à entrer en contact avec les étudiants français du cours sans avoir l'impression de déranger. De même, comme la gestion des projets communs s'est avérée être problématique, travaux en groupe et gestion des projets menés par plusieurs étudiants feront partie du cours de préparation également.

La dimension interculturelle de la préparation à la mobilité internationale est essentielle (Jackson, 2011; Papatsiba, 2003) et sera intégrée dans l'ensemble des cours que nous préparons. Il est important de familiariser les étudiants avec les concepts de l'interculturel, des différences interculturelles et avec l'adaptation dans un pays étranger (Bennet, 2013; Fitzpatrick, 2020; Ting-Tommey \& Dorjee, 2019). C'est pourquoi des exemples concrets des malentendus ou problèmes seront étudiés et analysés avec les étudiants sous forme d'études de cas.

Grâce à la participation des étudiants Erasmus français ou francophones en mobilité à Brno, les étudiants de l'université tchèque pourront discuter avec les étudiants Erasmus étrangers sur les différences perçues, sur les stéréotypes, clichés, habitudes ou règles qui peuvent être différents d'un pays à l'autre (retards, pauses, rafraîchissements en classe). Cette discussion permettra aux étudiants tchèques et slovaques de voir leur propre culture à travers les yeux des personnes venant d'ailleurs. Aussi d'autres difficultés identifiées grâce à notre étude pourraient être travaillées : abréviations, langue des sms, langue des jeunes (activité de traduction des messages ou textos, par exemple) ou la peur d'initier une interaction avec d'autres personnes.

D'autres thématiques du cours de préparation à la mobilité seront ajoutées plus tard, puisque le recueil des données de notre recherche continue, même pendant 
l'année académique 2020/2021 ce qui permettra de compléter notre recherche avec de nouvelles spécificités de la mobilité internationale dans les conditions de la pandémie actuelle. Les étudiants actuellement en mobilité, tout comme les 5 étudiantes du printemps 2020, sont à l'épreuve de l'adaptation aux restrictions sanitaires qui changent et ont besoin d'être flexibles et de gérer l'imprévisible.

Nous planifions d'élargir l'échantillon des personnes interviewées à des employés des universités - enseignants ou employés administratifs des universités françaises ou francophones pour pouvoir comparer et contraster les données sortantes de notre étude, mais aussi apporter le point de vue des établissements d'accueil.

La partie sur la comparaison des systèmes universitaires français ou francophones et tchèques sera enrichie par les informations données grâce aux entretiens avec les étudiants Erasmus français qui arrivent en séjour Erasmus à l'Université Masaryk de Brno. Nous avons déjà réalisé 6 entretiens semi-directifs depuis 2018, et continuons à ajouter d'autres répondants à cette recherche, y compris dans l'année universitaire actuelle de 2020/2021 ce qui permettra de contraster les différentes expériences d'études pendant la crise covid-19 et la vie des étudiants étrangers en France et en République tchèque.

\section{Conclusion}

L'analyse des entretiens nous a permis de connaître les types et le déroulement des interactions auxquelles les répondants ont participé pendant leur mobilité d'études. Grâce aux résultats de cette analyse, les enseignants de français de l'université d'origine pourraient adapter le contenu et les activités d'enseignement de manière à mieux préparer leurs étudiants à un tel séjour dans un pays francophone et les mener à gérer au mieux les difficultés éventuelles qui ressortent des interactions réalisées.

En élargissant l'échantillon des répondants, nous pourrons poursuivre notre recherche sur le sujet puisque la pandémie du Covid 19 a entraîné de nouvelles situations de communication. Une partie des étudiants sont, malgré tout, toujours intéressés par la mobilité Erasmus et la situation actuelle ne les a pas dissuadés de partir dans une université étrangère. Il serait donc intéressant de voir comment leurs besoins évoluent au fur et à mesure des changements dus aux restrictions sanitaires dans tous les domaines de la vie étudiante. Les résultats de la recherche pourront également servir aux étudiants qui souhaiteraient effectuer leur mobilité internationale mais sont découragés par l'inconnu et ont peur de l'imprévisible. 


\section{Bibliographie}

BENNET, M. J. (2013). Basic concepts of intercultural communication. Paradigms, principles and practices. Boston : Nicholas Brealy.

Bordo, W., GoEs, J. \& MANGiANTE, J.-M. (2016). Le français sur objectif universitaire. Entre apports théoriques et pratiques de terrain. Arras : Artois Presses Université.

CAVAlla, C. (2010). Méthodologie d'apprentissage de l'écrit universitaire, Le français dans le monde, recherche et applications, 47, p. 153-161.

Dervin, F. \& Byram, M. (2008). Échanges et mobilités académiques. Quel bilan ? Paris : L'Harmattan.

FitZPATRICK, F. (2020). Understanding Intercultural Interaction. An Analysis of Key Concepts. Bingley : Emerald Publishing.

GoEs, J. \& MANGiANTE, J.-M. (2010). Les écrits universitaires : besoins linguistiques et méthodologiques des étudiants allophones, Le français dans le monde, Recherche et applications, 47, p. 142-152.

JaCKson, J. (2011). Host Language Proficiency, Intercultural Sensitivity, and Study Abroad. In The Interdisciplinary Journal of Study Abroad. (21). p. 167-188.

Mangiante, J.-M. \& PARPETte, C. (2011). Le Français sur objectif universitaire. Grenoble : PUG.

Murphy-Lejeune, E. (2013). L'étudiant européen voyageur, un nouvel étranger. Paris : Didier.

Papatsiba, V. (2003). Des étudiants européens. Erasmus «et l'aventure de l'altérité». Berne : Peter Lang.

PARPette, C. \& Stauber, J. (2014). Réussir ses études d'économie-gestion en français. Grenoble : PUG.

Perez-Encinas, A., Rodriguez-Pomeda, J. \& DE WIT, H. (2020). Factors influencing student mobility : a comparative European study, Studies in Higher Education, p. 1-14.

Ting-ToomeY, S. \& DorJEe, T. (2019). Communicating across cultures. New York : Guilford Press.

\section{Auteur}

Mgr. Marie Červenková, Ph.D., e-mail : 18575@mail.muni.cz, ORCID https ://orcid.org/0000-00026639-5426, Language Centre, Masaryk University

Marie Červenková is a graduate of the Masaryk University in Brno. She has taught French in different types and levels of schools and is currently a lecturer at the Language Centre of the Faculty of Economics and Administration of Masaryk University in Brno, where she teaches Business French. In her work, she is interested in university teaching of foreign languages and in didactic and methodological questions associated with it (blended learning, flipped classes, developement of intercultural skills, specific vocabulary teaching).

Ing. Mgr. Hana Delalande, e-mail : delalande@ped.muni.cz, Department of French Language and Literature, Faculty of Education, Masaryk University

Hana Delalande is a teacher of Business French at Masaryk University and a PhD student (thesis topic : Development of Intercultural Communication Competence in French for Specific Purposes). She is interested in Intercultural Communication Competence, Professional mobility, Student mobility, Internships and Cooperation between universities and labour market. She studied Public Administration and Economy at Masaryk University, Brno, Czech Republic and University of Rennes (2009-2011) and Didactics of French and English at University of South Bohemia (2000-2005). 